\title{
Didaktische Qualifizierung der Lehrenden des Fachbereichs Finanzen der FH Bund (Münster)
}

\author{
Rainer Albrecht"
}

\section{A. Einleitung}

Der Beitrag informiert über ein didaktisches Qualifizierungsprogramm am Fachbereich Finanzen der Fachhochschule des Bundes. An diesem Programm nehmen aufgrund der Personalstruktur des Fachbereichs - überwiegend Juristinnen und Juristen $^{1}$ teil. Das Programm besteht aus einem obligatorischen, mehrteiligen Grundlagenmodul für die neuen Lehrenden und einem vielgestaltigen Vertiefungsmodul, an dem alle Lehrenden interessengeleitet teilnehmen können. Das Grundlagen- und Vertiefungsmodul weisen einen Gesamtumfang von jeweils 200 Stunden auf - wobei im Grundlagenmodul etwa die Hälfte der Stunden in Präsenz geleistet wird.

\section{B. Konzeptionelle Überlegungen}

\section{Selbstverständnis der Hochschuldidaktik}

Gegenstand der Hochschuldidaktik ist der Zusammenhang von Lehren und Lernen an Hochschulen. Dieser Zusammenhang wird durch zahlreiche Aspekte beeinflusst, die auf unterschiedlichen Ebenen reflektiert werden können: ${ }^{2}$

- Makroebene

Anthropogene Voraussetzungen (z.B. Erkenntnisse der Hirnforschung), gesellschaftliche Anforderungen, rechtliche Bestimmungen (z.B. Zugangsvoraussetzungen), Lehr- und Lernkultur, Prüfungswesen, institutionelle Ressourcen

- Mesoebene

Studien- und Lehrpläne, konzeptionelle Vorgaben für Module und Lehrveranstaltungen, Teilnahmevoraussetzungen, zeitliche und räumliche Rahmenbedingungen

- Mikroebene

Vorbereitung, Durchführung und Reflexion einzelner Unterrichtseinheiten bzw. einzelner Lehr-Lern-Interaktionen

In der Hochschuldidaktik werden diese Ebenen mit den drei Schlagworten „Personalentwicklung“ (Mikroebene), „Programmentwicklung“ (Mesoebene) und „Organisationsentwicklung“ (Makroebene) assoziiert, wobei die Personalentwicklung eingebettet im Kontext der anderen beiden Ebenen - im Zentrum hochschuldidaktischer Fortbildung steht.

* Dr. Rainer Albrecht ist Leiter des Fachgebiets Pädagogik am Bildungs- und Wissenschaftszentrum der Bundesfinanzverwaltung in Münster.

1 Aus Gründen der sprachlichen Vereinfachung und Lesbarkeit wird an zahlreichen Stellen im Text ausschließlich die männliche Form verwendet. Weibliche Personen sind jeweils mit angesprochen.

2 Wildt, in: Berendt/Wildt et al. (Hrsg.), S. 7 ff. 


\section{Was ist Gegenstand und Ziel didaktischer Fortbildung?}

In seiner jüngst auch in Deutschland intensiv diskutierten Metastudie konstatiert Hattie die herausragende Rolle der Lehrperson für das Gelingen von Lehr- und Lernprozessen. Die zentrale Schlussfolgerung lautet: „Lehrpersonen gehören zu den wirkungsvollsten Einflüssen beim Lernen. "3 Aus diesem Grunde dominiert der Aspekt der individuellen Kompetenzentwicklung die hochschuldidaktischen Fortbildungsprogramme zu Recht. ${ }^{4}$ Was kennzeichnet kompetente Lehrpersonen? ${ }^{5}$

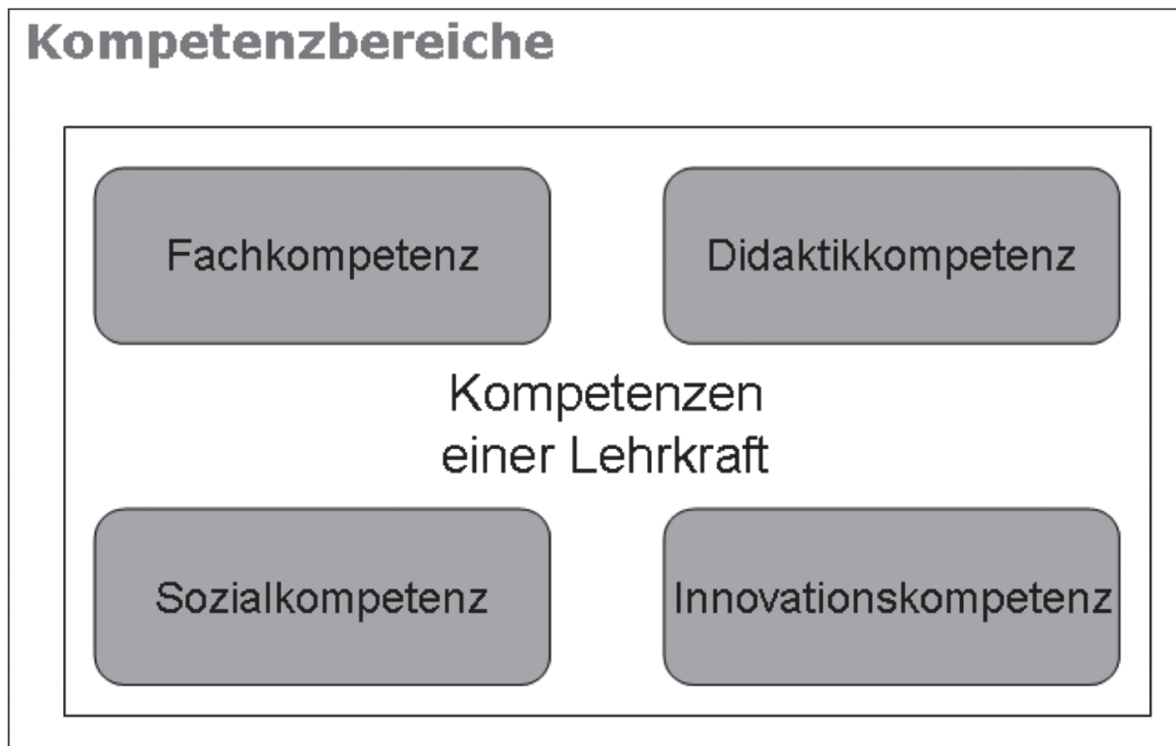

Abbildung 1: Kompetenz einer Lehrkraft

Fachkompetenz stellt eine Voraussetzung für Didaktikkompetenz dar. Hiermit sind Wissensbestände über wissenschaftliche Erkenntnisse und Methoden des Faches gemeint. Darüber hinaus ist es erforderlich, auch über Wissen (und Erfahrung) zu verfügen, um das jeweilige Fach auch ,gut' unterrichten zu können. Beispielsweise indem der Erklärungswert einzelner Inhalte für die jeweiligen Adressaten richtig eingeschätzt werden kann.

Sozialkompetenz bezieht sich auf den Umgang mit Lernenden, aber auch auf den Kontakt mit Kollegen. Lehren ist eine Tätigkeit, die in einem sozialen Rahmen in

3 Hattie, Lernen sichtbar machen, S. 280. Einen aktuellen Überblick zu den Erkenntnissen der Hirnforschung - und deren Bedeutung für die Pädagogik - bietet Roth, in: Zeitschrift für Pädagogische Psychologie 3 (2013), S. $123 \mathrm{ff}$.

4 Zahlreiche Beiträge über hochschuldidaktische Fortbildungsprogramme finden sich in: Berendt/Wildt et al. (Hrsg.).

5 Riedl/Schelten, Grundbegriffe der Pädagogik und Didaktik, S. 131 f. 
Form zwischenmenschlicher Interaktion erfolgt. In vielen Lehrsituationen muss im sozialen Handeln die richtige Balance zwischen konsequentem und unterstützendem Verhalten gefunden werden.

Das Erfordernis der Innovationskompetenz weist darauf hin, dass sich unsere Wissensgesellschaft in permanenter Veränderung befindet. Entsprechend daraus resultierender veränderter gesellschaftlicher Anforderungen befindet sich das Bildungssystem in einem unablässigen Anpassungsprozess. Eine fortlaufende Erneuerung eigener Lehrveranstaltungen ist hier nur die logische Folge.

Didaktikkompetenz kennzeichnet die Fähigkeit, aus der Vielzahl der Möglichkeiten jeweils passende Lerngegenstände auszuwählen und diese adressatengerecht darzustellen und zu bearbeiten. Sie umfasst aber auch die Verbindung „theoretischer“ Kenntnisse über Lehren und Lernen (z.B. Wissen über die Phasen des Lernprozesses) mit „praktischen“ Fähigkeiten und Fertigkeiten (z.B. die praktische Gestaltung einer Lehrveranstaltung). Entscheidend bei dieser Verbindung ist, dass die sich aus unterschiedlichen Quellen (Wissen und praktische Erfahrung) speisende Kompetenz nur (weiter-)entwickelt werden kann, wenn Planung, Durchführung und Reflexion des eigenen Lehrhandelns einen Regelkreis bilden.

\section{Didaktikkompetenz}

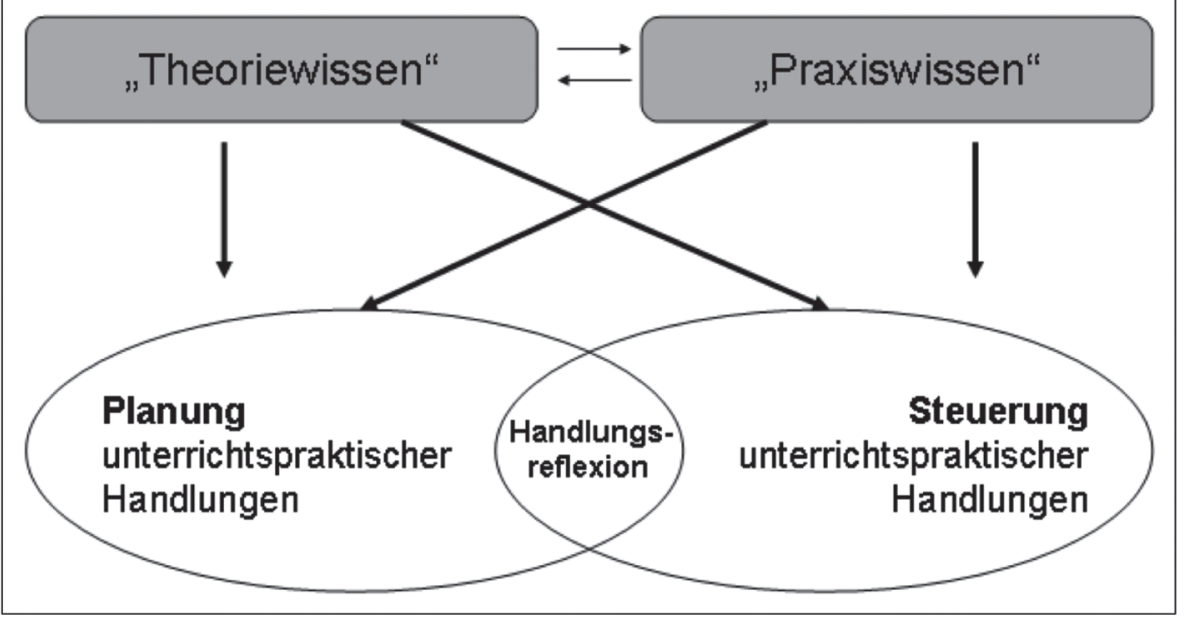

Abbildung 2: Reflexionskompetenz als Schlüssel für die Entwicklung der Didaktikkompetenz ${ }^{6}$

6 Riedl, Didaktik der beruflichen Bildung, S. $21 \mathrm{f}$. 
Insbesondere der Stellenwert praktischer Erfahrungen und instrumenteller Kompetenzen in Verbindung mit der Notwendigkeit einer systematischen Reflexion der eigenen Praxis hat eine wichtige zeitliche Dimension: Die Entwicklung von Didaktikkompetenz stellt einen individuellen Prozess dar, der i.d.R. über mehrere Jahre verläuft. ${ }^{7}$ Durch hochschuldidaktische Fortbildung soll dieser Prozess gezielt unterstützt werden. Die Wahl des Zeitpunkts geeigneter Fortbildungsmaßnahmen stellt allerdings ein gewisses Problem dar: Erfolgen sie zu früh (bevor eigene Lehrerfahrungen gemacht werden konnten), fehlt ein zentraler Bezugspunkt der Kompetenzentwicklung. Erfolgen sie zu spät, besteht die Gefahr, dass die individuelle didaktische Kompetenz ausschließlich aus Praxiswissen resultiert, das dann nicht ausreichend reflektiert (und daher auch nicht weiterentwickelt) werden kann.

Typischerweise vollzieht sich die Kompetenzentwicklung, wie üblich bei komplexeren Anforderungen, ${ }^{8}$ in mehreren Stufen:

\section{Entwicklung didaktischer Kompetenzen}

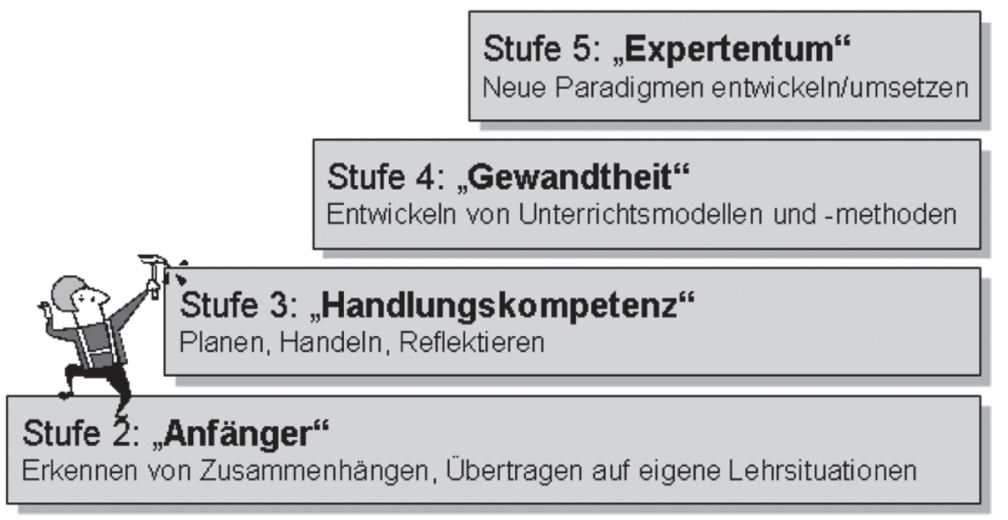

Stufe 1: „Neuling“

Wiedererkennen, Nachahmen, Erinnern

Abbildung 3: Adaption des DREYFUS-Modells der Kompetenzstufen auf die Domäne der Didaktikkompetenz

Idealerweise sollte hochschuldidaktische Fortbildung also in einer Phase stattfinden, in der die Stufe „Neuling“ bereits überwunden wurde und dann mit dazu beitragen, dass die Stufe „Anfänger“ möglichst zügig bewältigt werden kann und für die weitere

7 Jank/Meyer, Was ist guter Unterricht?, S. 134.

8 Das Modell der Entwicklung von Kompetenzen in fünf typischen Stufen wurde von dem amerikanischen Philosophen Dreyfus beschrieben. In Deutschland wurde dies in einer Arbeit über die Grenzen künstlicher Intelligenz veröffentlicht (vgl. ders., Künstliche Intelligenz). 
persönliche Entwicklung ein gutes Fundament mit der Stufe „Handlungskompetenz“ vorhanden ist. Es geht also um die planmäßige Unterstützung des gelingenden Übergangs von Stufe 2 auf 3.

\section{Grundstruktur hochschuldidaktischer Fortbildung}

Aufgrund der bisherigen konzeptionellen Vorüberlegungen folgt die Bearbeitung hochschuldidaktischer Fortbildungsthemen einer typischen Ablaufstruktur:

- Sensibilisierung für das Thema durch Aufzeigen der Relevanz für Lehren und Lernen

Beispiel: Die Sozialform,Gruppe' bietet ein wichtiges Potential für Lernprozesse. Die Erschließung dieses Potentials ist aber an Voraussetzungen gebunden. Oft gelingen Lernprozesse in Gruppen nicht bzw. das Verhältnis von (Zeit-)Aufwand und Lernergebnis stehen aus Lehrenden- und/oder Lernendensicht in keinem günstigen Verhältnis.

- Aktivierung des Vor- bzw. Erfahrungswissens

Beispiel: In welchen Situationen haben Sie (als Lehrperson aber auch als Teilnehmerin bzw. Teilnehmer) gute Erfahrungen mit Gruppenarbeiten gemacht? Was geht bei Gruppenarbeiten häufig schief?

- Darstellung bzw. Erarbeitung relevanter Theorien bzw. Modelle

Beispiel: Darstellung von Erkenntnissen zum Thema ,Gruppenverluste' (Sozialpsychologie). Erarbeitung eines idealtypischen Ablaufschemas von Gruppenarbeit (Didaktik/Methodik).

- Verarbeitung der neuen Informationen durch Abgleich mit dem Vorwissen bzw. mit der bisherigen Praxis

Beispiel: Die Teilnehmenden verarbeiten das neue Wissen, indem versucht wird, die bisherigen Praxiserfahrungen unter Berücksichtigung der theoretischen Konzepte zu erklären: „Warum ging es bei mir schief? “ bzw. „Warum hat es bei mir (trotzdem) geklappt? “

- Transfer dieser Überlegungen auf künftige eigene Lehrveranstaltungen, zunächst in konzeptioneller Hinsicht

Beispiel: Planung und Ausarbeitung einer Gruppenarbeit für eine eigene Lehrveranstaltung.

- Reflexion dieser Konzepte durch Diskussion in der Lerngruppe

Beispiel: Die Planung wird in der Seminargruppe vorgestellt und diskutiert. Ggf. wird die Vorbereitungsphase im Rahmen einer Lehrsimulation vorgeführt.

- Praktische Umsetzung in eigenen Lehrveranstaltungen

Beispiel: In einer eigenen Lehrveranstaltung wird die ausgearbeitete Gruppenarbeit durchgeführt.

- Reflexion der eigenen Praxis (mit einem Mentor bzw. innerhalb der Fortbildungsgruppe)

Beispiel: Verlauf und Ergebnisse der durchgefübrten Gruppenarbeit werden mit einem Mentor (der ggf. sogar hospitiert hat) bzw. in der nächsten Seminarphase in der Lerngruppe besprochen. 
Es wird hierbei deutlich: Wenn alle Schritte dieser Ablaufstruktur berücksichtigt werden sollen, kann eine systematische Unterstützung der Kompetenzentwicklung nicht allein durch einzelne Workshops oder Lehrgänge geleistet werden. Notwendig wäre eine mehrteilige Veranstaltungsreihe, die auch Phasen der praktischen Lehrtätigkeit mit einschließt. Durch ein die Praxisphasen begleitendes Mentoring kann die Reflexionskompetenz gezielt erweitert werden.

\section{Besonderheiten des Münsteraner Fortbildungsprogramms}

Der Fachbereich Finanzen der Fachhochschule des Bundes weist einige organisationale Besonderheiten auf, die hier kurz erwähnt werden sollen:

- Durch die Einbettung des Fachbereichs in das Bildungs- und Wissenschaftszentrums der Bundesfinanzverwaltung sind viele der Teilnehmenden auch bzw. sogar ausschließlich als ,hauptamtlich Lehrende' in der Fortbildung tätig. Die didaktische Fortbildung kann sich also nicht nur auf Lehrveranstaltungen an einer Fachhochschule konzentrieren, sondern muss auch den Bereich der beruflichen Fortbildung mit in den Blick nehmen.

- Die Lehrenden sind Bundesbeamte in einer dem Bundesfinanzministerium (BMF) nachgeordneten Behörde. Das BMF hat per Erlass geregelt, dass die hauptamtlich Lehrenden des Bildungszentrums ein sog. pädagogisches Feststellungsverfahren erfolgreich absolvieren müssen. Dieses stellt die formelle Abschlussprüfung des Grundlagenmoduls dar. Der erfolgreiche Besuch der didaktischen Fortbildung stellt also eine notwendige Voraussetzung dar, um dauerhaft am Bildungszentrum zu lehren.

- Neben diesem obligatorischen Grundlagenmodul, das alle Lehrenden des Bildungszentrums absolvieren, existiert seit drei Jahren ein Vertiefungsmodul, das allen Lehrenden die Möglichkeit bietet, interessegeleitet an einer didaktischen Fortbildung teilzunehmen. Hierbei werden größtenteils Themen vertieft, die aus zeitlichen Gründen im Grundlagenmodul nur gestreift werden können.

\section{Ablaufstruktur des Grundlagenmoduls}

Drei einwöchige Fortbildungsveranstaltungen (im Gesamtumfang von 99 Lehrveranstaltungsstunden) sind in Praxisphasen mit mentorieller Begleitung eingebettet, damit ausreichend Gelegenheit zur praktischen Erprobung besteht. Die Prüfung („Feststellungsverfahren“) erfolgt i.d.R. spätestens drei Monate nach der letzten Präsenzwoche im Rahmen einer realen Lehrveranstaltung mit Reflexion: 


\begin{tabular}{|l|}
\hline $\begin{array}{l}\text { Praxisphase I (30 LVS) } \\
\text { Hospitation und Teamteaching }\end{array}$ \\
\begin{tabular}{|l|}
\hline Seminarphase I (33 LVS) \\
Grundlagen des Lehrens und Lernens
\end{tabular} \\
\hline $\begin{array}{l}\text { Praxisphase II (8 Wochen) } \\
\text { Angeleitetes Unterrichten (12 LVS + Hospitationen) }\end{array}$ \\
\begin{tabular}{|l|}
\hline Seminarphase II (33 LVS) \\
Unterrichtspraktisches Handeln
\end{tabular} \\
\begin{tabular}{|l|} 
Praxisphase III (8 Wochen) \\
Selbständiges Unterrichten (12 LVS + Hospitationen)
\end{tabular} \\
\begin{tabular}{|l} 
Seminarphase III (33 LVS) \\
Reflexion eigenen Handelns und eigener Unterrichtskonzeptionen
\end{tabular} \\
\hline $\begin{array}{l}\text { Feststellungsverfahren (2 LVS) } \\
\text { Etwa 1-3 Monate Vorlauf, um Anregungen erproben zu können }\end{array}$ \\
\hline
\end{tabular}

Abbildung 4: Phasen des Grundlagenmoduls im Überblick

\section{Funktion und thematische Schwerpunkte der Seminarphasen}

Die Seminarphasen im Grundlagenmodul dienen überwiegend der fachwissenschaftlichen Auseinandersetzung mit relevanten Themen. ${ }^{9}$ Bereits vorhandenes (Erfahrungs-)Wissen soll systematisch um pädagogisches, didaktisches und psychologisches Fachwissen erweitert werden. Damit diese Fachwissenschaften (Pädagogik/Didaktik und Psychologie) in allen Aspekten der Fortbildung berücksichtigt werden können, werden die Seminarphasen im Teamteaching durchgeführt. Die Themen der Seminarphasen sind in vier Cluster gegliedert:

- Lernpsychologie

Lernbegriff, Modelle der Lernpsychologie, Biologische Grundlagen des Lernens, Phasen des Lernprozesses, Lernen als Wissenserwerb, Motivationstheoretische Grundlagen, Bedingungen für Lernmotivation, Lerntypen, Motivationsquellen in Lehrveranstaltungen

- Didaktische Planung

Analyse der Teilnehmenden, Zielanalyse/Formulierung aussagekräftiger Feinziele, Sachanalyse/Stoffreduktion, Lehrpläne und Dokumentation eigener Veranstaltungen (Didaktische Analyse und Verlaufsplanung)

- Lehren und Lernen als soziales Handeln

9 Im Gegensatz dazu weisen die Veranstaltungen des Vertiefungsmoduls überwiegend einen Trainingscharakter auf. 
Eigene Rolle als Lehrperson, Kommunikation im Unterricht, Persönlichkeitspsychologie, Umgang mit Heterogenität, Gruppendynamik, Störungen und Konflikte, Umgang mit schwierigen Teilnehmenden

- Unterrichtsmethoden und -medien

Grundlegende Unterrichtsverfahren (darbietend, erarbeitend und gesprächsorientiert), Sozialformen des Unterrichts (Einzel-, Partner- und Gruppenarbeit), Lehrmedien und ihre jeweiligen Einsatzmöglichkeiten, Lehrvorträge, Einsatz von Schriftmedien, Lehrgespräche, Anleiten und Auswerten von Übungen, aktivierende Lehrmethoden, Gruppenarbeit

In der ersten Seminarwoche werden schwerpunktmäßig die ersten beiden Themencluster (Lernpsychologie und Didaktische Planung) bearbeitet. Hiermit soll erreicht werden, dass zu Beginn wichtige Grundbegriffe und theoretische Konzepte verfügbar gemacht werden und mit diesen anwendungsbezogen im Laufe der Fortbildung weiter gearbeitet werden kann.

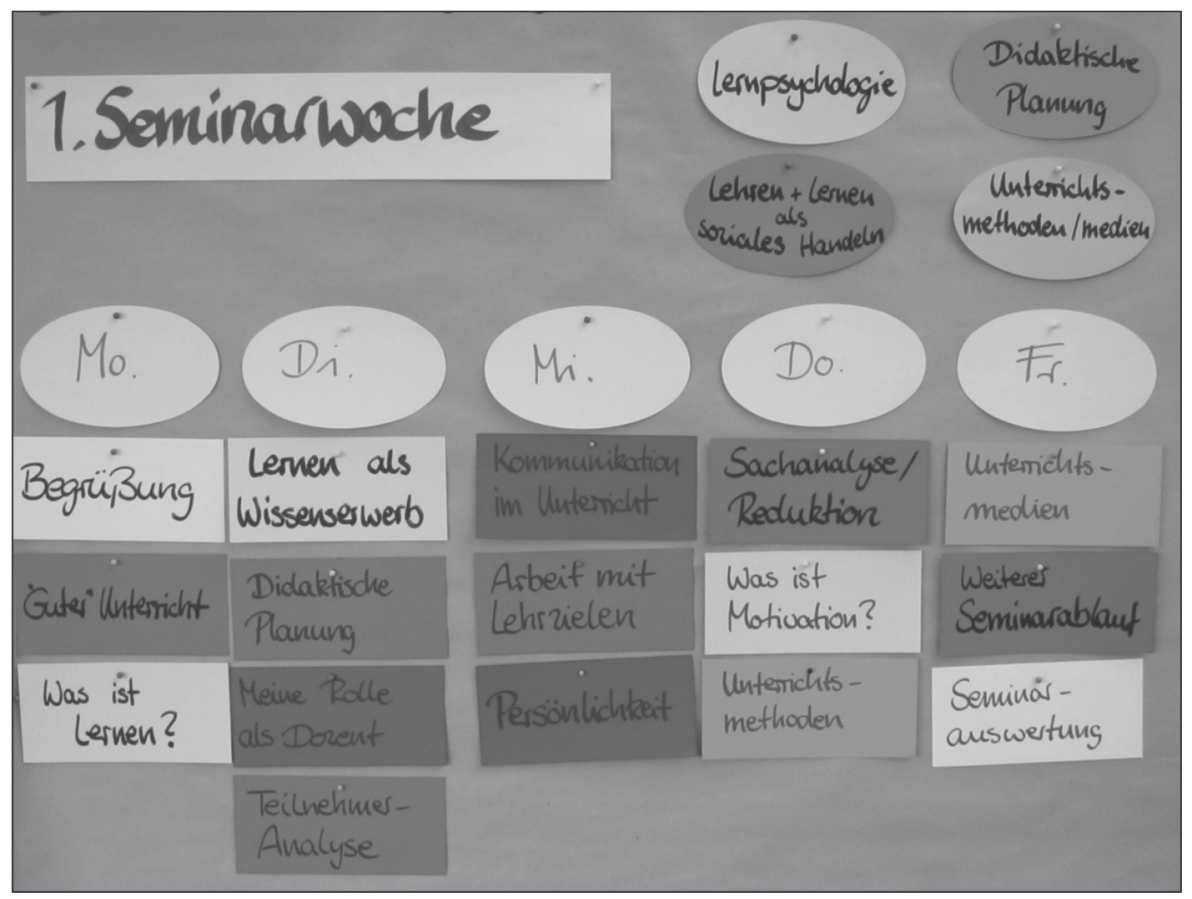

Abbildung 5: Themen der ersten Seminarwoche

In der zweiten Seminarwoche steht das unterrichtspraktische Handeln im Vordergrund: Einsatz und Gestaltung von Unterrichtsmedien und das methodische Handeln in Lehrveranstaltungen bieten vielfältige Möglichkeiten für eine Bearbeitung in der oben dargestellten Form. 
Die dritte Seminarwoche stellt die Lehrveranstaltungskonzepte der Teilnehmenden in den Mittelpunkt: Diese arbeiten im Vorfeld das didaktische Konzept einer Lehrveranstaltungsstunde schriftlich aus und führen einen (etwa 20-minütigen) Ausschnitt daraus im Rahmen einer Lehrsimulation vor. Die Lehrsimulation und das Konzept werden ausführlich besprochen. Hiermit werden vor allem zwei Funktionen erfüllt: Die Teilnehmenden erhalten zu zahlreichen Aspekten ihrer Planung und praktischen Durchführung Rückmeldungen aus der Seminargruppe. Gleichzeitig üben sich die Teilnehmenden in der gezielten Beobachtung relevanter Aspekte und deren konstruktiver Rückmeldung an Ihre Kolleginnen und Kollegen. Bei Bedarf und soweit zeitlich möglich - können auch einzelne Aspekte der bisherigen Seminarthemen weiter vertieft werden.

\section{Funktion und thematische Schwerpunkte der Praxisphasen}

Wie oben dargestellt, sind die drei Seminarwochen in Phasen der Lehrpraxis eingebettet. Möglichst sollten erste Erfahrungen mit der Rolle des Lehrenden bereits vorliegen, bevor die Teilnahme an der ersten Seminarveranstaltung erfolgt. Wenn dies nicht im Rahmen eigener Lehrveranstaltungen geschehen kann (weil beispielsweise aus organisatorischen Gründen noch keine eigenen Lehr- bzw. Fortbildungsveranstaltungen stattfinden), soll dies durch Teamteaching bzw. Hospitationen im Umfang von mindestens 30 Lehrveranstaltungsstunden gewährleistet werden. Die Praxisphasen werden mentoriell begleitet. Das heißt, für jeden neuen Lehrenden wird noch vor Seminarbeginn ein Mentor benannt. Dieser soll folgende Voraussetzungen erfüllen: Der Mentor soll

- über mehrjährige Lehrerfahrung am BWZ verfügen.

- nicht Vorgesetzter des neuen Lehrenden sein.

- den neuen Lehrenden in seiner persönlichen Entwicklung als Lehrenden unterstützen.

- zur Aufgabenübernahme bereit sein.

- möglichst immer nur einen Mentee begleiten.

- bereit und in der Lage sein, zusätzlich zu den Zeitaufwendungen für das Mentoring einmal jährlich an einer zweitägigen Fortbildungsveranstaltung für Mentoren teilzunehmen.

Der Mentor hospitiert regelmäßig in den Lehrveranstaltungen des neuen Lehrenden und gibt diesem konstruktive Rückmeldungen. Vor dem jeweiligen Unterrichtsbesuch wird im Rahmen eines Vorgesprächs festgelegt, welche Themen im Zentrum der Rückmeldung stehen. Auf diese Weise kann der neue Lehrende selbst besser steuern, welche Seminarthemen in der praktischen Umsetzung schwerpunktmäßig behandelt werden.

Außerdem erhalten die Lehrgangsteilnehmer Beobachtungsaufträge, die im Rahmen der Hospitation bei anderen (erfahrenen) Lehrenden bearbeitet werden sollen. Diese Beobachtungsaufträge knüpfen thematisch an die jeweils zurückliegende Seminarphase an. Sie sollen dazu anregen die Seminarinhalte im Hinblick auf praktische 
Relevanz zu überprüfen. Zu jedem Beobachtungsauftrag wird ein Beitrag in einem Diskussionsforum verfasst, das den Teilnehmenden exklusiv auf einer E-LearningPlattform zur Verfügung steht. Auch der weitere Austausch und die Information der Teilnehmenden werden während der Praxisphasen über diese Lernplattform organisiert.

In der ersten Praxisphase - also noch vor der ersten Seminarwoche - sollen die Teilnehmenden in einer Lehrveranstaltung hospitieren und ihre Beobachtungen zu folgenden Aspekten im Rahmen eines Forumsbeitrags schildern: ${ }^{10}$

- Methodische Grundstruktur von Unterricht (Einstiegs-, Arbeitsphase, Phase der Ergebnissicherung)

- Methodische Gestaltungsmöglichkeiten der einzelnen Unterrichtsphasen

- Rolle des Dozenten (Verantwortungsbereiche, Selbstverständnis)

In der zweiten Praxisphase sollen möglichst viele der zuvor behandelten Seminarthemen mit praktischen Erfahrungen abgeglichen werden - entweder in Form von Umsetzungsversuchen in eigenen Lehrveranstaltungen oder durch gezielte Beobachtungen im Rahmen von Unterrichtshospitationen. Der Mentor soll an mindestens einer Lehrveranstaltung des neuen Lehrenden beobachtend teilnehmen und seine Beobachtungen in einer strukturierten Form an den neuen Lehrenden zurückmelden. Außerdem soll er für mindestens drei weitere Beratungsgespräche zur Verfügung stehen.

In der dritten Praxisphase sollen erneut die in den Seminarphasen behandelten Themen mit Erfahrungen in den eigenen Lehrveranstaltungen verbunden werden. Hierzu werden Beobachtungsaufträge für Hospitationen bei anderen Lehrenden (beispielsweise zum Thema Störungen, zur Gruppendynamik oder zur Ausgestaltung spezieller Methoden) erteilt. Auch soll der Mentor an einer - möglichst selbständig entwickelten - Unterrichtseinheit des neuen Lehrenden teilnehmen. In dieser Unterrichtseinheit sollen einige der zuvor behandelten Themen möglichst modellhaft umgesetzt werden. Die Beobachtungen des Mentors sollen in einer strukturierten Form an den neuen Lehrenden zurückgemeldet werden.

In der Regel findet das Feststellungsverfahren erst mehrere Wochen nach dem letzten Seminar statt. Diese Zeit sollte genutzt werden, um die Anregungen aus der letzten Seminarphase gezielt umzusetzen. Hierbei unterstützt der Mentor durch teilnehmende Beobachtungen und Beratungsgespräche.

\section{Prüfung („pädagogisches Feststellungsverfahren“)}

Die Teilnahme am Grundlagenmodul endet mit einer förmlichen Prüfung (dem sog. pädagogischen Feststellungsverfahren), an der neben der Seminarleitung auch die Leitung des jeweiligen Studienbereichs bzw. Fachgebiets als Vorgesetzter des neuen Lehrenden sowie jeweils ein weiterer erfahrener Lehrender eines anderen Studien-

10 Die Teilnehmenden erhalten konkrete Fragestellungen; an dieser Stelle werden aus Platzgründen nur die Themen benannt. 
bereichs bzw. Fachgebiets beteiligt sind. Das Feststellungsverfahren besteht aus einer schriftlichen Unterrichtsvorbereitung (Didaktische Analyse und Verlaufsplanung), der Durchführung einer Lehrveranstaltungsstunde mit realen Teilnehmern, an dem die Feststellungskommission beobachtend teilnimmt, sowie in der Durchführung eines anschließenden Reflexionsgesprächs. Zur Beurteilung der Probelehrveranstaltung und des Reflexionsgesprächs wurde ein Katalog mit Bewertungskriterien entwickelt. ${ }^{11}$ Dieser wird nicht quantitativ ausgewertet, sondern dient den Kommissionsmitgliedern zur Unterstützung einer strukturierten Beobachtung und Beratung.

\section{Beurteilung der Probelehrveranstaltung}

Unterrichtsstruktur

$$
\begin{array}{cc}
\text { trifft } \mathrm{zu} & \text { trifft } \\
\text { nicht } \mathrm{zu}
\end{array}
$$

Die Planung der Unterrichtseinheit berücksichtigt ausreichend Phasen, in denen die für die $\mathrm{TN}^{12}$ neuen Informationen durch Übungen (oder andere geeignete Aktivitäten) von den TN verarbeitet werden können.

Der Unterricht gliedert sich erkennbar in einzelne Phasen:

Einstiegs-, Informations-, Arbeitsphase und Phase der Ergebnissicherung.

Die einzelnen Phasen sind inhaltlich und organisatorisch aufeinander bezogen und bauen sinnvoll aufeinander auf.

Der Zeitbedarf für die einzelnen Unterrichtsphasen wird realistisch eingeschätzt.

Die Einstiegsphase informiert über Ziele und Verlauf der Stunde. In der Informationsphase werden die für die TN neuen Informationen nach didaktischen Kriterien dargestellt bzw. von den TN erarbeitet. In der Phase der Ergebnissicherung werden die wichtigsten Ergebnisse der Stunde zusammengefasst und ein Bezug zu nachfolgenden Stunden ggf. auch zu anderen Fächern aufgezeigt.

\section{Teilnehmerorientierung}

Der Zeitbedarf für die einzelnen Unterrichtsphasen wird flexibel dem Leistungsvermögen der TN angepasst.

Der Umgang mit den TN ist durch Wertschätzung geprägt.

Der Unterricht bietet Elemente um die Motivation der TN zu befördern bzw. aufrecht zu erhalten.

Vorkenntnisse und Erfahrungen der TN werden berücksichtigt. Wo es möglich ist, können die TN diese in den Unterricht einbringen.

Der Unterricht berücksichtigt unterschiedliche Verarbeitungs- und Beteiligungsmöglichkeiten der TN.

11 Die Kriterien repräsentieren den Stand der hausinternen Diskussion zum Thema ,gute Lehre' bzw. ,guter Fachunterricht'. Die besonderen Rahmenbedingungen des Bildungszentrums (Aus- und Fortbildung, Gruppengröße i.d.R. max. 25 Personen) wirken sich hier entsprechend aus.

12 Teilnehmenden. 


\section{Methodische und mediale Gestaltung}

trifft zu trifft nicht

$\mathrm{zu}$

Die methodische Gestaltung des Unterrichts begründet sich aus der Didaktischen Planung: Ziele, Inhalte, TN-Voraussetzungen und Rahmenbedingungen.

Die Unterrichtsgestaltung ist am Prinzip der Aktivierung orientiert: Die TN werden im Unterrichtsverlauf (z.B. durch Fragen, Impulse, Arbeitsaufträge, Übungen) so oft wie möglich aktiviert. Den TN wird dadurch erleichtert eine aktive Lernhaltung einzunehmen.

Der/die Lehrende motiviert die TN Fragen zu stellen und geht in angemessener Weise auf Fragen ein; auch indem diese aufgegriffen, umformuliert, weitergegeben oder zurückgestellt werden.

Der/die Lehrende formuliert selbst motivierende Fragen und Aufgaben.

Die Medienwahl ist an didaktischen Prinzipien orientiert und leistet einen Beitrag Zusammenhänge für die TN nachvollziehbarer, verständlicher bzw. anschaulicher zu machen.

Die Gestaltung der Medien wird den Gegebenheiten, den TN und dem dargestellten Gegenstand gerecht.

\section{Reflexion der Probelehrveranstaltung und des Unterrichtskonzepts}

Das eigene Handeln und das der TN kann realistisch beschrieben trifft zu trifft nicht $\mathrm{zu}$ werden.

Das eigene Handeln kann - auch unter Berücksichtigung der eigenen Unterrichtskonzeption (Didaktische Analyse und Verlaufsplanung) begründet werden.

Das Handeln der TN kann - auch unter Hinzuziehung didaktischer und psychologischer Modelle und Konzepte - interpretiert werden. Abweichungen von der eigenen Planung, z.B. im Hinblick auf methodische Aspekte oder Zeitmanagement, werden vom Lehrenden wahrgenommen.

Die wahrgenommenen Abweichungen werden vom Lehrenden schlüssig interpretiert.

Alternative Vorgehensweisen im Hinblick auf die Unterrichtskonzeption und das methodische Handeln können vom Lehrenden benannt und diskutiert werden.

Die Kommission entscheidet mit einfacher Mehrheit über die pädagogische Eignung. Die Entscheidung wird umgehend mitgeteilt und begründet. Im Falle einer negativen Entscheidung darf das Feststellungsverfahren einmal wiederholt werden. 


\section{Themen des Vertiefungsmoduls}

Die Vertiefungsveranstaltungen haben einen Umfang von zwei bis drei Tagen und überwiegend Trainingscharakter. Der Gesamtumfang beträgt derzeit etwa 200 Stunden. Die Inputphasen sind vergleichsweise kurz. Die Zeit soll hauptsächlich für das praktische Erproben und dessen Reflexion/Bewertung zur Verfügung stehen. Derzeit werden folgende Veranstaltungen wiederkehrend - ein- bis zweimal jährlich - angeboten:

\begin{tabular}{|l|}
\hline Vertiefungsveranstaltungen \\
\begin{tabular}{|l|}
\hline Stimmtraining (Teil 1 und 2) \\
Gezielter Einsatz von Stimme und Körpersprache in der Lehre \\
\hline $\begin{array}{l}\text { Gesprächsführung } \\
\text { Fragend-entwickelnde Gesprächsführung im Unterricht }\end{array}$ \\
\hline $\begin{array}{l}\text { Schwierige Situationen (Zwei Seminarvarianten) } \\
\text { Umgang mit Störungen und Konflikten }\end{array}$ \\
\hline $\begin{array}{l}\text { Methodenwerkstatt } \\
\text { Aktivierende Methoden in Lehrveranstaltungen }\end{array}$ \\
\hline $\begin{array}{l}\text { Visualisieren (Teil 1 und 2) } \\
\text { Veranschaulichen von Lehrinhalten auf Flipchart }\end{array}$ \\
\hline $\begin{array}{l}\text { Vortragstechnik } \\
\text { Gestaltung interessanter und informativer Lehrvorträge }\end{array}$ \\
\hline $\begin{array}{l}\text { Fachdidaktische Vertiefung } \\
\text { Wissenschaft \& Technik; IT-Lehrgänge; Trainings }\end{array}$ \\
\hline
\end{tabular} \\
\hline
\end{tabular}

Abbildung 6: Veranstaltungen des Vertiefungsmoduls

1. Gezielter Einsatz von Stimme und Körpersprache in Lehrveranstaltungen Basisseminar ${ }^{13}$

Erfolgreiches Auftreten, überzeugendes Agieren und stimmliche Souveränität sind keine angeborenen Fähigkeiten. Sie können erlernt werden. Als Grundlage des Seminars wird die Funktionsweise von Stimme und Körpersprache besprochen, um diese gezielt einsetzen zu können. Darauf aufbauend werden die wichtigsten Prinzipien zum Vortragen praktisch geübt. Über die Schulung von Eigen- und Fremdwahrnehmung während der Übungen bekommen die Teilnehmenden ein direktes Feedback zu ihrer eigenen Wirkung und Präsenz. Es wird sowohl in der Großgruppe als auch in zwei Kleingruppen gearbeitet, um noch effektiver auf die verschiedenen

13 Bei den folgenden Veranstaltungsbeschreibungen handelt es sich um leicht gekürzte Fassungen aus dem aktuellen Veranstaltungsprogramm. 
Bedürfnisse einzugehen. Im Anschluss erhalten alle Teilnehmenden eine nachfassende E-Mail mit individuellem Feedback, Tipps und Übungen.

\section{Gezielter Einsatz von Stimme und Körpersprache in Lehrveranstaltungen - Aufbauseminar}

Die individuelle Wirkung als Lehrperson kann durch den gezielten Einsatz von Stimme und Körpersprache erheblich verbessert werden: Durch packendes und authentisches Auftreten sowie stimmliche Überzeugungskraft gewinnt die persönliche Präsenz in Aus- und Fortbildungsveranstaltungen. Im Aufbaukurs stehen sowohl die Vertiefung der Vortrags- und Präsentationstechniken als auch weiterführende Übungen für Stimme und Körpersprache im Vordergrund. Mit Hilfe von Videoanalysen kann die Eigen- und Fremdwahrnehmung verbessert werden, die eigene Entwicklung wird sichtbar. Alle Teilnehmenden erhalten eine Video-CD der eigenen Vorträge sowie ein individuelles Feedback.

- Verbesserung der Präsenz und der Wirkung auf die Zuhörer

- (Selbst)bewussteres Auftreten, dadurch mehr Authentizität

- Optimierung der Vortragstechniken und Kommunikationsfähigkeiten

- Ökonomisierung des Stimm- und Körpereinsatzes

\section{Gesprächsführung in Aus- und Fortbildungsveranstaltungen}

Das Gespräch in Lehrveranstaltungen gibt uns viele Möglichkeiten an die Hand, unsere Teilnehmenden in das Geschehen einzubeziehen: Vorwissen kann eingebracht, Unklares erfragt, Strittiges diskutiert werden. Durch die Beteiligung möglichst vieler am Gespräch erhalten wir wichtige Rückmeldungen und Anregungen für die weitere Unterrichtsgestaltung. Gespräche sind also für ,guten' Unterricht enorm wichtig. Was aber kennzeichnet effektive Unterrichtsgespräche? Welche Gesprächsformen sind praktikabel und welche (unterschiedlichen) Wirkungen sind mit ihnen verbunden? Zentral sind die ,richtigen' Fragen bzw. Impulse und wie wir mit Reaktionen umgehen. Einen Schwerpunkt der Veranstaltungen bilden die Gestaltungselemente des sog. fragend-entwickelnden Unterrichtsgesprächs und wie diese Gesprächsform in eigenen Lehrveranstaltungen gewinnbringend eingesetzt werden kann.

- Verschiedene Gesprächsformen in Aus- und Fortbildung unterscheiden können

- Begründen des spezifischen Nutzens einzelner Gesprächsformen in eigenen Lehrveranstaltungen

- Das fragend-entwickelnde Lehrgespräch in eigenen Lehrveranstaltungen einsetzen können

\section{Kollegiale Fallberatung zur Bearbeitung schwieriger Lehrsituationen in der Lehre}

Störungen und Konflikte kommen in allen Lehr- und Fortbildungsveranstaltungen immer wieder vor. Typische Beispiele hierfür sind:

- Fortlaufendes Zuspätkommen einzelner Teilnehmer/-innen

- Fortlaufende Nebengespräche oder Nebentätigkeiten (Email) 
- Ablehnung bestimmter Arbeitsformen (Gruppenarbeit, Hausarbeit)

- Durch Häufigkeit oder Inhalt von Wortbeiträgen sehr dominante Teilnehmer/-innen

- Verweigerung der Mitarbeit

- Konflikte innerhalb der Lerngruppe bzw. zwischen Lerngruppe und Dozent/-in

Die Fortbildungsveranstaltung orientiert sich an von den Teilnehmern eingebrachten „schwierigen“ Situationen. Mit der Methode der kollegialen Fallberatung wird gemeinsam erarbeitet, wie sich manche Situation von vornherein vermeiden ließe, wie reagiert werden kann, wenn es zu Problemen kommt und wie diese möglichst „unspektakulär" beendet bzw. bearbeitet werden können.

- Welche Situationen in Lehrveranstaltung erlebe ich persönlich als schwierig bzw. stressig?

- Welche Ursachen sind möglicherweise dafür verantwortlich?

- Wie kann ich durch Veränderung meiner didaktischen Konzeption bzw. durch mein Lehrverhalten problematische Situationen von vornherein vermeiden?

- Wie reagiere ich konstruktiv auf meine „schwierigen“ Situationen?

\section{Schwierige Situationen in Lehrveranstaltungen}

Im Lehralltag können unterschiedliche Störungen und Konfliktsituationen auftreten: es gibt Disziplinprobleme, Studierende verweigern (scheinbar) die Mitarbeit oder greifen in Diskussionen Dozierende oder andere Studierende an. In diesem Workshop werden typische Konfliktsituationen bearbeitet und individuelle Lösungsstrategien entwickelt.

- Eigenen Umgang mit störenden Situationen/Stress reflektieren

- Ursachen für typische Störungen in eigenen Lehrveranstaltungen erkennen

- Vorbeugende Maßnahmen für typische Störungen in eigenen Lehrveranstaltungen entwickeln

- Interventionstechniken konstruktiv einsetzen können

\section{Methodenwerkstatt: Aktivierende Methoden in Aus- und Fortbildungsveranstaltungen}

Effektiv Lehren heißt auch: Lernen lassen! Die aktive Teilhabe der Lernenden an Lehr- und Fortbildungsveranstaltungen ist für den Lernerfolg von entscheidender Bedeutung. Doch wie kann diese ,aktive Teilhabe' so organisiert werden, dass sie zielorientiert verläuft und außerdem im vorgegebenen Zeitrahmen leistbar ist?

Spezielle - aktivierende - Methoden können hier hilfreich sein. Zunächst geht es darum, die bekanntesten dieser Methoden (auch durch praktisches Ausprobieren) kennen zu lernen. Im zweiten Schritt wird geprüft, in welcher Weise welche Methoden in eigene Aus- und Fortbildungsveranstaltungen integriert werden könnten. Auf diese Weise werden Anregungen dazu gegeben, das eigene Methodenrepertoire systematisch zu erweitern. 
- Methodeneinsatz in Aus- und Fortbildungsveranstaltungen unter didaktischen Gesichtspunkten planen können

- Kenntnis der wichtigsten aktivierenden Methoden

- Integration aktivierender Methoden in eigene Lehr- bzw. Fortbildungsveranstaltungen

- Erweiterung des eigenen Methodenrepertoires

\section{Wirkungsvoll visualisieren mit Flipchart in Aus- und Fortbildungsveranstaltungen}

Eine wichtige Kernkompetenz von Trainern und Dozenten ist die Visualisierung von Lehrinhalten. Visualisierungen sind hilfreich, um Informationen lebendig und strukturiert zu transportieren, Seminarteilnehmer aktiv einzubeziehen und Ergebnisse nachhaltig zu sichern. Vor allem der Einsatz von Bildern ist dabei bedeutend: Sie bereichern und erweitern die Kommunikation, sie geben anregende Impulse in Lernprozesse und erreichen Menschen auf einer anderen Ebene als nur über Sprache.

In diesem Seminar wird vermittelt, wie Visualisierungen (vor allem an Flipchart oder Pinnwand) gekonnt gestaltet und wirkungsvoll genutzt werden können. Inhalte werden auf das Wesentliche reduziert und in eine sinnvolle Struktur und ansprechende Gestaltungsform gebracht; eine visualisierungsgerechte Schrift sowie die gezielte Kombination von Farben und Formen unterstützen die Verständlichkeit. Mit einfachen, aber effektvollen, skizzenhaften Darstellungen wird „Wortsprache“ mit „Bildsprache" ergänzt.

Es wird praktisch: Die Teilnehmenden werden an ihren konkreten Themen aus der Lehre arbeiten. Zeichnerisches Talent ist ausdrücklich keine Voraussetzung für die Teilnahme an diesem Seminar.

- Lehrinhalte visuell ansprechend und sinnvoll strukturiert aufbereiten

- Skizzenhaftes Zeichnen: Tipps und Tricks für schnelle, reduzierte Darstellungen

- Vom Thema zum Bild: Übersetzung von Inhalten in eine Bildsprache

- Visualisierungs-Varianten kennenlernen und für die eigene Berufspraxis umsetzen

- Visualisierung und bildhafte Darstellung in Lernprozessen gezielt einsetzen und zur Teilnehmeraktivierung nutzen

- den eigenen Lehr- und Präsentationsstil erweitern

\section{Werkstatt Visualisieren - Aufbauseminar}

Lehrende, die durch ihre Teilnahme am Basisseminar über die Grundlagen der Visualisierung verfügen, finden hier einen „kreativen Freiraum“, in dem Sie ihre Themen visualisieren können. Die Seminarleitung coacht und gibt weitere Anregungen, die bei der Umsetzung unterstützen. In kleinen Übungseinheiten werden verschiedene Techniken vertieft. Die Teilnehmenden profitieren vom gegenseitigen Ideenaustausch und Feedback.

\section{Lehrvorträge in Aus- und Fortbildungsveranstaltungen}

Der Vortrag ist ein wichtiger Bestandteil vieler Lehrveranstaltungen. Speziell wenn die Teilnehmenden noch sehr wenig über das zu bearbeitende Thema wissen, profi- 
tieren alle vom Vortrag: Durch diese Unterrichtsmethode ist es möglich, komplexe Zusammenhänge in strukturierter Form und kurzer Zeit anschaulich darzustellen. In dieser Fortbildungsveranstaltung geht es um Gestaltungsmöglichkeiten, die einen ,guten' Vortrag ausmachen: Themenwahl, Gliederung, Stimulanz, Anschaulichkeit (auch im Zusammenwirken mit Präsentationsmedien) und die Interaktion mit den Zuhörern.

- Vorträge teilnehmer- und nutzenorientiert aufbauen

- Formulieren motivierender Vortragstitel und -einstiege

- Komplexe Sachverhalte verständlich darstellen

- Zusammenspiel mit Präsentationsmedien

- Interaktion mit den Teilnehmenden

\section{Schlussbetrachtung}

Das vorgestellte Programm zur didaktischen Qualifizierung zeigt beispielhaft auf, wie die Entwicklung didaktischer Kompetenzen von Lehrenden an einer Hochschule systematisch unterstützt werden kann.

Die anspruchsvolle Prüfung am Ende des Grundlagenmoduls spielt - insbesondere im Vergleich mit anderen Programmen - eine wichtige Rolle: Die Zielperspektive des Grundlagenmoduls wird dadurch für alle Beteiligten transparent. Die hausinterne Diskussion der Bewertungskriterien befördert den fortlaufenden Austausch über die Kriterien guter Lehre. Dies wird auch durch den Umstand unterstützt, dass alle Lehrenden das Seminar absolviert haben. Hierdurch wird faktisch ein Standard gesetzt, der von einer breiten Basis getragen wird. Die Motivation der neuen Lehrenden ist fast ausnahmslos sehr hoch. Die Anmeldungen zu den Veranstaltungen des Vertiefungsmoduls sind aktuell dreimal so hoch wie Teilnahmeplätze zur Verfügung stehen.

Sehr günstig wirkt sich der Umstand aus, dass das Grundlagenmodul aus mehreren, aufeinander aufbauenden Elementen besteht. Die Teilnahme erfolgt innerhalb des ersten Jahres zur Hauszugehörigkeit in einer festen Seminargruppe. Die hierbei sich vollziehende Gruppenbildung unterstützt die Teilnehmenden in ihrer individuellen Kompetenzentwicklung noch zusätzlich.

Die systematische Berücksichtigung der Lehrpraxis ist ein weiterer wichtiger Faktor, der entscheidend zur Wirkung des Grundlagenmoduls beiträgt. Auf diese Weise kann nicht nur für die Teilnehmenden der individuelle Transfer erleichtert werden. Auch für die konzeptionelle Weiterentwicklung des Programms ist gesorgt, denn die Qualität der Seminarphasen wird durch die fortlaufenden Rückmeldungen aus der Praxis automatisch evaluiert.

\section{Literaturverzeichnis}

Berendt, Brigitte/Wildt, Johannes/Szczyrba, Birgit (Hrsg.), Neues Handbuch Hochschullehre, Lehren und Lernen effizient gestalten, Stuttgart 2002. 
Dreyfus, Hubert L., Künstliche Intelligenz, Hamburg 1987.

Hattie, John, Lernen sichtbar machen, Baltmannsweiler 2013.

Jank, Werner/Meyer, Hilbert, Was ist guter Unterricht?, 5. Auflage, Berlin 2008.

Riedl, Alfred, Didaktik der beruflichen Bildung, Stuttgart 2004.

Roth, Gerhard, Welchen Nutzen haben die Erkenntnisse der Hirnforschung für die Pädagogik, in: Zeitschrift für Pädagogische Psychologie 3 (2013), S. 123-133.

Riedl, Alfred/Schelten, Andreas, Grundbegriffe der Pädagogik und Didaktik beruflicher Bildung, Stuttgart 2013.

Wildt, Johannes, Ein hochschuldidaktischer Blick auf Lehren und Lernen, in: Berendt/Wildt/Szczyrba (Hrsg.), Neues Handbuch Hochschullehre, Lehren und Lernen effizient gestalten, Stuttgart 2012, A 1.1, S. 1-10. 